\title{
Bilateral Pneumonia in a Patient with Chronic Bronchiectasis Caused by Achromobacter xylosoxidans Subspecies denitrificans
}

\author{
Gauthier Stepman ${ }^{1}$, Kulveer Dabb ${ }^{1}$, Imran A. Khan ${ }^{2}$, Jordan T. Young ${ }^{1}$, Johnathan Frunzi ${ }^{1}$ \\ 1. Internal Medicine, Medical Center of Trinity, Trinity, USA 2. Pulmonology, Medical Center of Trinity, Trinity, USA
}

Corresponding author: Gauthier Stepman, gauthier.stepman@hcahealthcare.com

\begin{abstract}
Achromobacter xylosoxidans is a gram-negative bacillus that has a multitude of inherent and acquired antimicrobial resistance. It is a rare, isolated pathogen in patients without cystic fibrosis (CF). We report the case of a 76-year-old Caucasian male with a history of chronic obstructive pulmonary disease (COPD), previous Mycobacterium-avium intracellulare (MAI) infection, and chronic bronchiectasis who did not respond to three courses of outpatient antibiotics for a chronic cough. He also had a 21-lb weight loss. The diagnosis of Achromobacter xylosoxidans subspecies denitrificans was made through bronchoscopy with bronchoalveolar lavage (BAL).
\end{abstract}

There are few case reports describing Achromobacter xylosoxidans subspecies denitrificans in non-CF patients. Achromobacter xylosoxidans colonization might be linked to predisposing lung damage such as in $\mathrm{CF}$ and bronchiectasis. The bacterium is frequently multidrug-resistant. More studies are needed to develop recommendations for clinical guidelines to address the increasing antibiotic resistance to Achromobacter xylosoxidans.

Received 02/27/2020 Review began 03/15/2020 Review ended 03/20/2020 Published 03/23/2020

\section{(c) Copyright 2020}

Stepman et al. This is an open access article distributed under the terms of the Creative Commons Attribution License CC-BY 4.0., which permits unrestricted use, distribution, and reproduction in any medium, provided the original author and source are credited.
Categories: Internal Medicine, Infectious Disease, Pulmonology

Keywords: achromobacter, xylosoxidans, denitrificans, bronchiectasis, pneumonia

\section{Introduction}

Achromobacteria are gram-negative, motile bacilli that are oxidase-positive, catalase-positive, and lactose nonfermenting [1-2]. They are often found in aquatic environments and soil [1]. The bacterium was first described by Yabuuchi and Ohyama in 1971, when it was isolated from otic discharge [3]. Several subspecies have been identified since its discovery in 1971, of which most are nonpathogenic [1-2]. Some subspecies, however, can cause significant disease in patients with predisposing tissue damage such as in cystic fibrosis (CF) or bronchiectasis. The most clinically significant subspecies are xylosoxidans and denitrificans $[2,4]$. It is thought that bacteremia with this organism is most likely to occur nosocomially and more specifically in immunocompromised patients [5]. One study found in a 10-year follow-up that solid organ cancers and heart failure were most commonly associated with Achromobacteria (A.) xylosoxidans bacteremia [6]. Pulmonary infections, however, are independent of a patient's immunocompetency but are believed to be related to lung tissue integrity $[5,7]$.

A. xylosoxidans subspecies denitrificans is less commonly reported as a pathogen than the xylosoxidans subspecies $[2,4]$. A. xylosoxidans is a relatively rare pathogen to cause respiratory disease but is more commonly found in patients with CF [8]. The reported prevalence rate of colonization in CF populations varies between $5.3 \%$ and $13.1 \%$ [8-9]. This case report describes a patient with a past medical history of chronic bronchiectasis who was diagnosed with pneumonia caused by $A$. xylosoxidans subspecies denitrificans.

\section{Case Presentation}

Our patient is a 76-year-old Caucasian male with a past medical history significant for chronic obstructive pulmonary disease (COPD), pulmonary Mycobacterium avium-intracellulare (MAI) infection, and bronchiectasis. He presented to his pulmonologist's office with complaints of persisted productive cough, subjective fevers, rhinitis, and a 21-lb unintentional weight loss over a period of three months. The patient was started on a seven-day course of doxycycline. However, doxycycline did not improve his cough. He was then prescribed a second course of doxycycline by his primary care physician, again without any clinical improvement. After completion of the second course of antibiotics, he was prescribed cefdinir $300 \mathrm{mg}$, twice daily for seven days. Due to the continued symptoms after his third antibiotic course, he was admitted to the hospital for further evaluation. During his hospitalization, a computed tomography (CT) scan was performed, which showed evidence of bronchiectasis (Figure 1) and bilateral lower lobe consolidations (Figure 2). 


\section{Cureus}

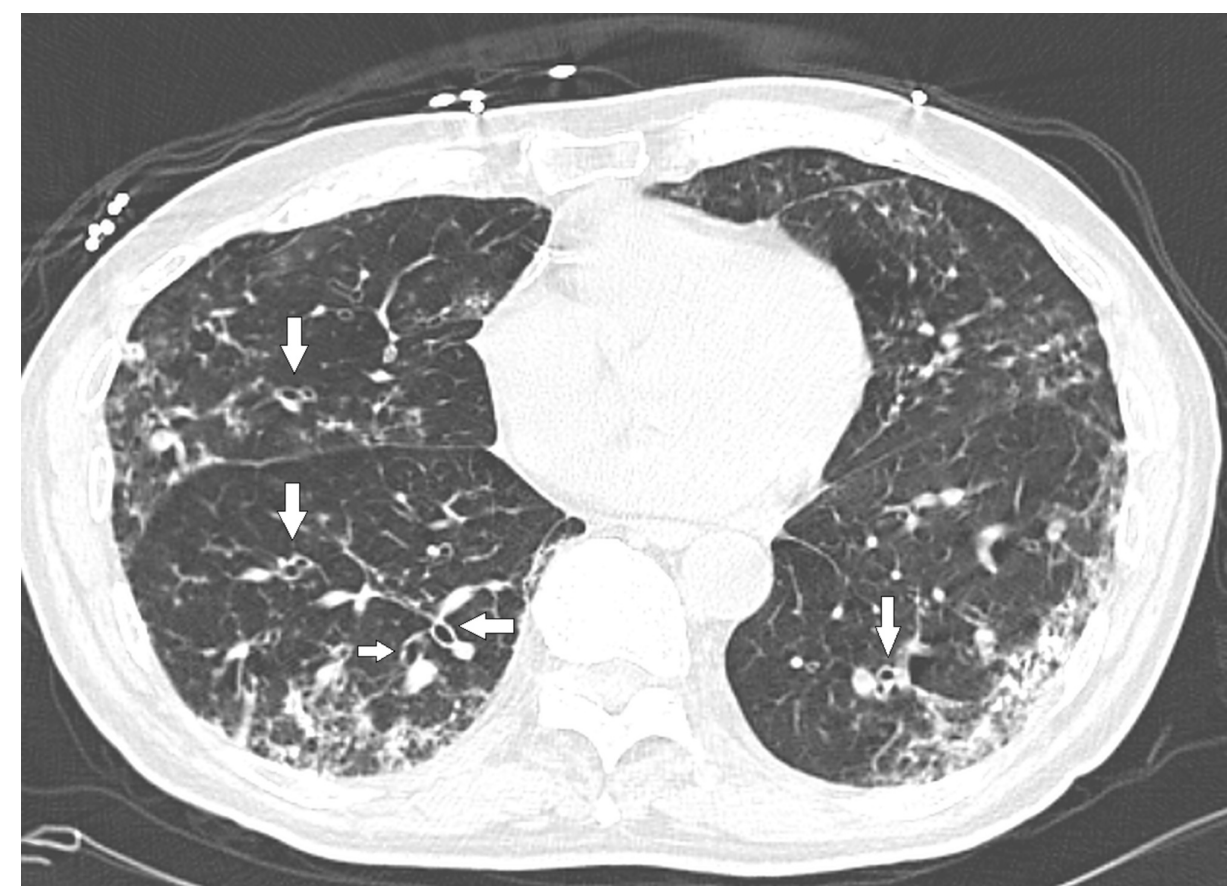

FIGURE 1: Computed tomography scan showing bronchiectasis

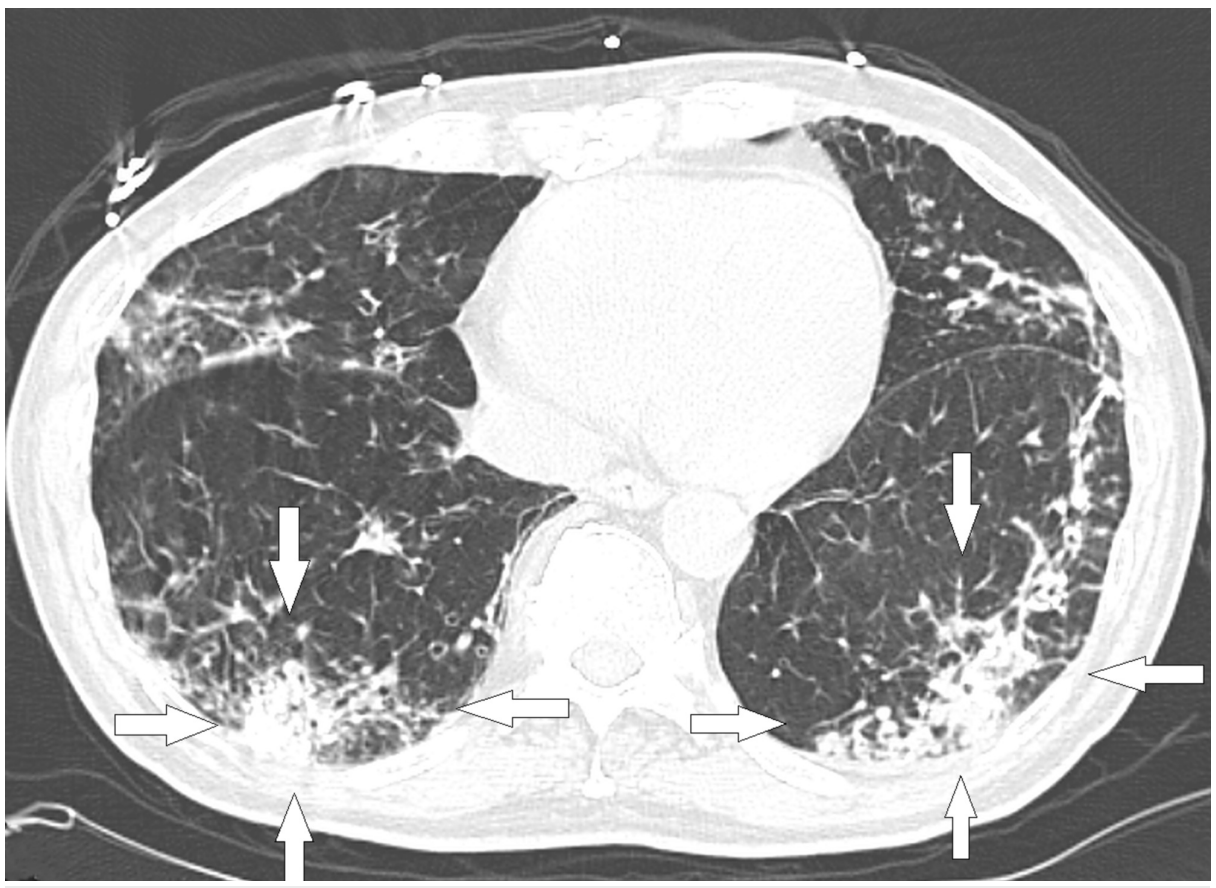

FIGURE 2: Computed tomography scan showing bilateral lower lobe consolidation

He was started on intravenous (IV) ceftriaxone 1 gram daily and oral azithromycin $500 \mathrm{mg}$ daily. Given his history of MAI infection and current prolonged history of coughing despite multiple courses of antibiotics, the patient was evaluated for immunodeficiency. Laboratory tests for immunoglobulins revealed normal to above-normal levels (see Table 1). 


\section{Cureus}

\begin{tabular}{|c|c|c|}
\hline Immunoglobulin & Level (mg/dL) & Reference Range (mg/dL) \\
\hline $\lg G$ & 1997 & $700-1600$ \\
\hline IgG1 & 1072 & $248-810$ \\
\hline $\lg \mathrm{g} 2$ & 500 & $130-555$ \\
\hline IgG3 & 104 & $15-102$ \\
\hline $\lg$ G4 & 105 & $2-96$ \\
\hline $\lg A$ & 461.9 & $70-400$ \\
\hline $\lg M$ & 48 & $40-230$ \\
\hline
\end{tabular}

\section{TABLE 1: Immunoglobulin levels in our patient}

Culture of the bronchoalveolar lavage revealed the presence of A. xylosoxidans subspecies denitrificans in both lower lobes. The lavage was also sent for acid-fast bacilli, which confirmed a recurrence of MAI. Antibiotic sensitivity tests for the A. xylosoxidans subspecies denitrificans strain in this patient is documented in Table 2 .

\begin{tabular}{|c|c|c|}
\hline Antimicrobial agent & Presence of sensitivity & MIC \\
\hline Gentamicin & Intermediate & 8 \\
\hline Tobramycin & Sensitive & 4 \\
\hline Ciprofloxacin & Resistant & $>=4$ \\
\hline Ceftazidime & Intermediate & 16 \\
\hline Amikacin & Sensitive & 16 \\
\hline Cefepime & Resistant & $>=64$ \\
\hline Piperacillin/Tazobactam & Sensitive & $<=4$ \\
\hline Meropenem & Sensitive & 1 \\
\hline
\end{tabular}

TABLE 2: Microbiological sensitivities of isolated Achromobacter species

The patient's antibiotic regimen was switched to IV meropenem due to the sensitivity test results. A peripherally inserted central catheter (PICC) was inserted and the patient was discharged with a two-week course of IV meropenem. The patient was seen two weeks after discharge by his primary care physician and five weeks after discharge by his pulmonologist. He reported feeling better with his cough being improved. He also reported some weight gain since his last visit.

\section{Discussion}

A. xylosoxidans is a rare cause of pneumonia in the immunocompetent adult. Infections with A. xylosoxidans, subspecies denitrificans are less common than with the xylosoxidans subspecies. Swenson and Sadikot reviewed 32 case reports of respiratory infections with A. xylosoxidans in patients without CF and found only three cases in which A. xylosoxidans, subspecies denitrificans was the causative organism [4]. We reviewed all current reported cases of pneumonia caused by A. xylosoxidans subspecies denitrificans and reported this in Table 3. 


\section{Cureus}

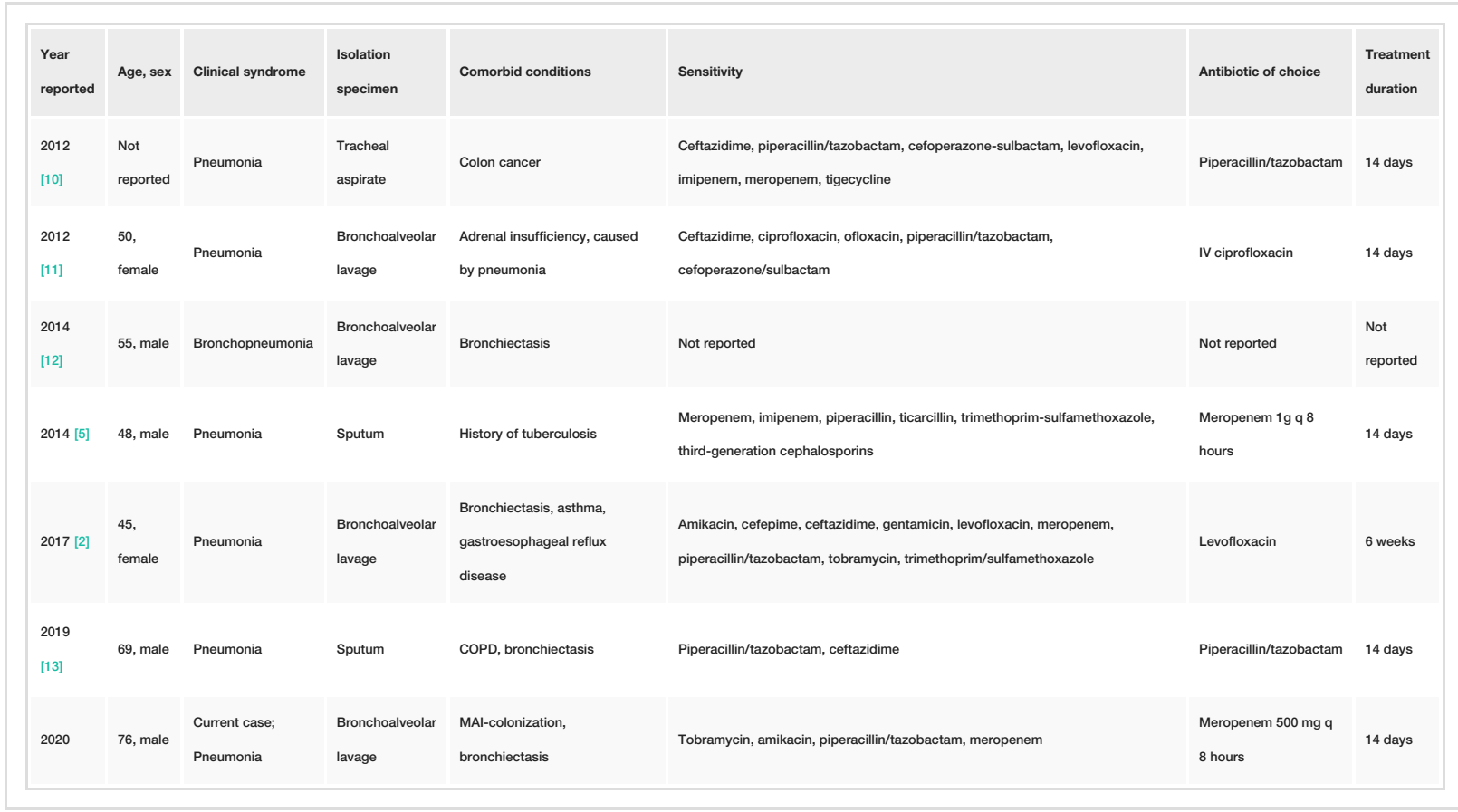

TABLE 3: Current case reports with Achromobacter xylosoxidans subspecies denitrificans as causative organism

IV: intravenous; COPD: chronic obstructive pulmonary disease; MAI: Mycobacterium avium-intracellulare

The presentation of A. xylosoxidans pneumonia is atypical. Common symptoms are fever, productive cough, weight loss, progressive shortness of breath, and fatigue $[2,5,14]$. The prolonged clinical course and resistance to multiple antibiotic treatments warrant further investigations.

Since A. xylosoxidans is most frequently associated with CF, it is believed that pre-existing lung damage makes patients more susceptible to colonization or infection with $A$. xylosoxidans $[4,6,8]$. Our patient had chronic bronchiectasis, most likely due to a previous MAI infection. This was similar to the patient described by Bharadiya et al., who had bronchiectasis secondary to previous mycobacterial infection [5]. In fact, most of the case reports that describe Achromobacter xylosoxidans, subspecies denitrificans pneumonia were in patients with bronchiectasis $[2,13-14]$.

Microscopically, A. xylosoxidans is very similar to Pseudomonas aeruginosa, which can potentially lead to lower rates of detection [8]. In patients with CF, the co-colonization of P. aeruginosa and A. xylosoxidans was associated with a significant decline in respiratory function [9]. Since patients with pre-existing lung damage are often colonized with $P$. aeruginosa, it was suggested to systematically eradicate $A$. xylosoxidans, as is currently recommended for $P$. aeruginosa $[9,14]$. This could potentially prevent a decline in respiratory function in patients with non-CF $A$. xylosoxidans infections.

A. xylosoxidans is frequently a multidrug-resistant organism (MDRO). The multidrug resistance is broad, however, and not uniform between different isolates, making antibiotic choice challenging [4].

A 10-year case series of A.xylosoxidans bacteremia noted that most isolates were susceptible to meropenem and piperacillin or tazobactam [7]. This was also the case for our patient. Current guidelines for the treatment of A. xylosoxidans infections in patients with bronchiectasis are not available. The current treatment practices are mainly based on bronchiectasis and P. aeruginos $a$ treatment [13].

\section{Conclusions}

The purpose of this case report is to inform physicians that patients with pneumonia, resistant to multiple antibiotics, need further investigations to identify the causative organism. This is especially true for patients with predisposing lung tissue damage, as in bronchiectasis and CF. Due to the microbiological similarities, treatment practices currently rely on $P$. aeruginos $a$ infections. More studies are needed to form guidelines on management. Since A. xylosoxidans is an uncommon MDRO pathogen to cause disease in non-CF patients, patients often fail multiple outpatient antibiotic treatments. The need for intravenous antibiotics is high, making outpatient treatment difficult. The MDRO nature of A. xylosoxidans makes it important to timely identify the pathogen as to not further increase resistance, especially in patients with lung damage, who are 
at increased risk. More studies are also needed to evaluate the influence of Achromobacter xylosoxidans respiratory infections on the pulmonary function.

\section{Additional Information \\ Disclosures}

Human subjects: Consent was obtained by all participants in this study. Conflicts of interest: In compliance with the ICMJE uniform disclosure form, all authors declare the following: Payment/services info: All authors have declared that no financial support was received from any organization for the submitted work. Financial relationships: Gauthier Stepman, Kulveer Dabb, Imran A. Khan, Jordan T. Young, Johnathan Frunzi declare(s) None from HCA Healthcare and/or an HCA Healthcare-affiliated entity. This research was supported (in whole or in part) by HCA Healthcare and/or an HCA Healthcare-affiliated entity. The views expressed in this publication represent those of the author(s) and do not necessarily represent the official views of HCA Healthcare or any of its affiliated entities. Other relationships: All authors have declared that there are no other relationships or activities that could appear to have influenced the submitted work.

\section{References}

1. Liu C, Guo J, Yan W, et al.: Hospital-acquired pneumonia due to Achromobacter xylosoxidans in the elderly: A single-center retrospective study in Beijing. J Infect Dev Ctries. 2017, 11:1. 10.3855/jidc.8747

2. Awadh H, Mansour M, Aqtash O, Shweihat Y: Pneumonia due to a rare pathogen: Achromobacter xylosoxidans, Subspecies denitrificans. Case Rep Infect Dis. 2017, 2017:3969682. 10.1155/2017/3969682

3. Yabuuchi E, Ohyama A: Achromobacter xylosoxidans n. sp. from human ear discharge . Jpn J Microbiol. 1971, 15:477-481. 10.1111/j.1348-0421.1971.tb00607.x

4. Swenson CE, Sadikot RT: Achromobacter respiratory infections. Ann Am Thorac Soc. 2015, 12:252-258. 10.1513/AnnalsATS.201406-288FR

5. Bharadiya A, Mane M, Pawar S, Aundhakar S: Watch out! Pneumonia secondary to Achromobacter denitrificans. Ann Med Health Sci Res. 2014, 14:22-24.

6. Barragán EP, Pérez JS, Corbella L, Orellana MÁ, Fernández-Ruiz M: Achromobacter xylosoxidans bacteremia: clinical and microbiological features in a 10-year case series. Rev Esp Quimioter. 2018, 31:268273.

7. De Baets F, Schelstraete P, Van Daele S, Haerynck F, Vaneechoutte M: Achromobacter xylosoxidans in cystic fibrosis: prevalence and clinical relevance. J Cyst Fibros. 2007, 6:75-78. 10.1016/j.jcf.2006.05.011

8. Tetart M, Wallet F, Kyheng M, et al.: Impact of Achromobacter xylosoxidans isolation on the respiratory function of adult patients with cystic fibrosis. ERS Monogr. 2019, 5:00051. 10.1183/23120541.00051-2019

9. Apau O, Farah N. Community-acquired pneumonia due to Achromobacter Denitrificans . (2019). Accessed: January 25, 2020: https://journal.chestnet.org/article/S0012-3692(19)32099-9/fulltext.

10. Chandrasekaran R, Mac Aogáin M, Chalmers JD, Elborn SJ, Chotirmall SH: Geographic variation in the aetiology, epidemiology and microbiology of bronchiectasis. BMC Pulm Med. 2018, 18:83. 10.1186/s12890018-0638-0

11. Atalay S, Ece G, Samlioğlu P, Kose S, Maras G, Gonullu M: Clinical and microbiological evaluation of eight patients with isolated Achromobacter xylosoxidans. Scand J Infect Dis. 2012, 44:798-801. 10.3109/00365548.2012.664780

12. Karanth Dr. SS, Gupta A, Prabhu M: Community acquired bilateral upper lobe pneumonia with acute adrenal insufficiency: a new face of Achromobacter xylosoxidans. Australas Med J. 2012, 5:531-533.

13. Community acquired Achromobacter bronchopneumonia. (2012). Accessed: February 2020: http://10.1164/ajrccm-conference.2014.189.1_MeetingAbstracts.A1748.

14. Bates AS, Natarajan M, Reddy RV: Achromobacter xylosoxidans in idiopathic cystic bronchiectasis. BMJ Case Rep. 2018, 11:e211610. 10.1136/bcr-2015-211610 